\title{
REFERRING TO WORLD OBJECTS WITH TEXT AND PICTURES
}

\author{
Elisabeth Andre, Thomas Rist \\ German Rescarch Center for Artificial Intelligence (DFKI) \\ D-66123 Saarbrücken, Germany, c-mail: \{andre, rist $\} @$ dfki.uni-sb.de
}

\begin{abstract}
ABSTRACI: It often makes sense to cmploy both text and pictures when referring to world objects. In this paper, we present a model for referring which is based on the assumption that concepts may be activated not only by $\mathrm{text}$, but also by pictures and text-picture combinations. By means of a case study, we demonstratc that failure and success of referring acts can be explained by the user's ability to infer certain links between mental representations and object descriptions. Irinally, we show how the model hats been incorporated into a plan-based multimedia presentation system by defining operators for concept activation.
\end{abstract}

\section{INTRODUCTION}

From a specen act theoretical point of vicw, referring is a planned action to achieve certain goals ( $\Lambda$ ppelt and Kronfeld, 1987). Although natural language may be the most conventional vehicic for referring, it has been widely accepted that pictures can be used as well. For cxample, Goodmann (1969) points out that pictures can be cmployed to refer to both an individual object and the type of which an object is an exemplary of. Morcover, there are good reasons 10 include pictures in referting acts. Pictures offectively convey discriminating object properties such as surface attributes and shape. If an object can only be discriminated against alternatives through its location, a picture may provide the spatial context of the object. Since depictions are cxplicit material representations of the world objects to which they correspond, new attributes of the typo 'being depicted as ...' are introduced which, in turn, provide an additional source for object discrimination (c.g., the knob which is represented by the black circle ...). Last but not Icast, scveral graphical focusing techniques can be applied to effectively constrain the set of alternatives (c.g., arrows, blinking). Unfortunately, there is also a dark side of the picture. An obvious drawback is that pictures do not provide for syntactical devices to distinguish between a reference-specifying and a predication-specifying part since objects and their propertics are hardly separable once depicted. Another difficulty is that pictures lack the means to distinguish definite from indefinite descriptions. Thus, it may remain unclear whether a particular object or whether an arbitrary exemplary of a class is depicted. The conclusion we can draw from these considerations is that it often makes sense to employ both text and pictures when referring to domain objects. Picures may be used in order to simplify verbal reference expressions. On the other hand, anbiguities of pictures can be resolved by providing additional information through text. When analyzing illustrated docuncnts such as assembly manuals and instructions for use, different kinds of referring expression can be found:

Multimedia referring expressions refer to world objects via a combination of at least two modia. Wach medium conveys some discriminating attributes which in sum allow for a proper identification of the intended object. Examples are NL expressions that are accompanicd by pointing gestures and text-picture combinations where the picture provides information about the appearance of an object and the text restricts the visual search space as in "the switch on the Crontside".

Anaphoric referring expressions refer to world objects in an abbreviated form (Hirst, 1981) presuming that they are already explicitly or implicitly introduced in the discourse. The prescntation part to which an anaphoric expression refers back is called the antecelent of the referring expression. In a multimedia discourse, we have not only to handle linguistic anaphora with linguistic anteccuents, but also linguistic anaphora with pictorial antecedents, and pictorial anaphora with linguistic or pictorial antecedents. Examples, such as "the hatched switch," show that the boundary betwecn multimedia referring expressions and anaphora is indistinct. Here, we have to consider whether the user is intended to cmploy all parts of a presentation for object disambiguation or whether one wants him to infer anaphoric relations betwecn them.

Cross-media referring expressions do not refer to world objects, but to document parts in other presentation mcdia (Wahlster et al, 1991). Lxamples of cross-modia referring expressions are "the upper left corner of the picture" or "Irig. $x "$ ". In most cases, cross-media referring expressions are part of a complex multimedia referring expresssion where they serve to direct the reader's attention to parts of a document that has also to be employed in order to find the intended referent.

When vicwing referring as a planned action, we have to specily which goals underly the use of different types of , referring cxpressions. Appelt and Kronfeld (1987) distin- guish between the literal goal and the discourse purpose of a reference act. Whereas the literal goal is to cstablish mutual belief between a speaker and a hearer that a particular object is being talked about, the discourse purpose is to make the hearer recognize what kind of identification is appropriate and to have him identify the referent accordingly. When addressing illustrated documents, the question arises of what identification means when domain objects ate referred to via piccures (and text). As with language this varics from discourse to discourse. For example, if the user is confronted with a picture showing how to insert the filter of a coffee machine, he has to recognize whether 


\begin{tabular}{|c|c|}
\hline $\begin{array}{l}\text { System } \\
\text { believes }\end{array}$ & 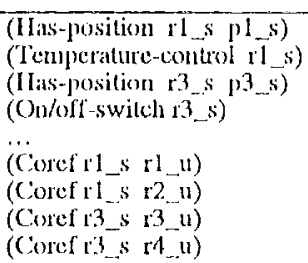 \\
\hline
\end{tabular}

\begin{tabular}{|c|c|}
\hline $\begin{array}{l}\text { System } \\
\text { believes } \\
\text { User } \\
\text { believes }\end{array}$ & 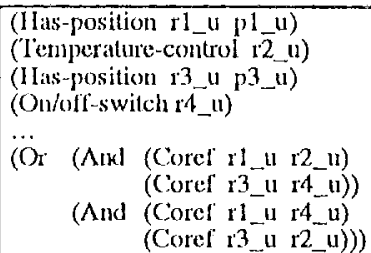 \\
\hline
\end{tabular}

Ir̈gure 1: Modelling I:xample: Different Knowledge Concening the Identity of Objects

any object with the feature 'being a filter' can be inserted or whether a particular object is meant. In the first case, he has to identify the picture object as an excmplary of a certain class whereas, in the sceond case, he has to look for something in the world which fits the graphical depiction. In olher siluations, identification involves cstabtishing a kind of colesive link between document parts. If the uset is confronted with a secpuence of pictures showing an object from different angles, he has to recognize that in all pictures the same object is depicted (pictorial anaphor with pictorial antecedent). When reading an utterance, such as "the resistor in the figure above," he has to recognize an anaploric relationship between the textual description and the graphical depiction (linguistic anapher with pictorial (antecedent).

Previous work on the generation of referring expressions in a multimodia cnvironment has mainly concentrated on single reference phenomenal, such as references to prictorial matcrial via natural language and pointing gestures ( $A 11$ gayer et al., 1989; (lasssen, 1992; Stock ct al., 1993) and the generation of cross-media references from tex l 10 graphlics (McKcown ct al., 1992; Wahtster et al., 1993). 'The aim of this paper is, however, to provide a more gencrat model that explains which kinds of cordcrential link between icferring expressions, objects of the world and objects of the multimedia presentation have to be established to cosure the comprehensibility of a referring expression.

\section{A MODEI, FOR REIERRING WITII TEXT AND IICIURLS}

When relering to domain objects a presentation systen has to find intelligible object descriptions which will activate appropriate representations. We assume that representations can be activated in the sense of picking then out of a set of representations which ate already available or which have to be buill up (c.g., by localizing an object in a user's visual ficld). Representations can be aclivaled by textual descriptions, by graphical descriptions or by mixed descriptions. Whereas the order in which representations are activated by a text is influcoed by the discourse structure, it is less than clear in which order a picture aclivates representations. If several objects are olepicted, the corresponding represcntations may be aclivated simultanconsly.

2.1 Representations of World Objects

To cosure the translerability of our approach, we don't presuppose a certain knowledge represcntation language. Ilowever, an essential part of the model concerns the distinclion between the system's beliefs about the world and the system's belict's about the user's beliefs. We represcnt llicse beliefs in different models. For example, the system may classify a certain object as an espresso machine while it assumes the user regateds the object as a coffec machine. lirthermore, we have to consider that the user's and the system's belichs about the identity of objects may differ. The system may believe that the user has different represcntations for onc and the same object without knowing how they are related to cach other. Conversely, it may happen that the user is assumed to have only one representation for objects which the system considers as distinct entities. As a consequence, our models can contain diflerent representations for one and the sane world object. We use the predicate

(Coref repl rep2)

to express that rep 1 and rep 2 are representations of the sane world object.

lig. 1 gives an example of how to use the concepts introduced above. let's start from the following situation taken from an espresso machine domain: The system knows that there are two switches (the temperature control and the on/oll switch) and also knows where they are located. Let I 1_s and $\mathrm{r} 3$.s correspond to the system's internal representations of the switches. The user is assumed to look at the espresso machine and to see two switches. I cet rl_u and r3.u correspond to interual representations of the switches which the user builds up when looking at the machine. We assume that the user also knows of the existence of the on/olf switch and the temperature control, but is not able to lecalize them. I ct $\mathbf{2}$.u and $\mathrm{r} 4$.u be the user's representations for the temperature control and the on/off switch. The lact that he only knows that one of the switehes he sees must be the temperature control and the other the on/off switch can be expressed by means of a disjunction. Bither a corel relation holds between $r 1_{-} u$ and $r 2 . u$ and between r3_u and r4_u or conversely, between r $L_{-} u$ and $r 4$ ru and between $r 3$.1 and ran. The connection between the system's representations il s and $\mathrm{r} 3 . \mathrm{s}$ to the representations the user is assumed to have is also expressed by coreference relations.

2.2 Representation of Descriptions

As mentioned in section 1, descriptions can be compesed 
of text, graphics and further presentation media. 'lo cope with such descriptions, we associate with cach syntactical unit (depictions, noun phrases, etc.) the set of object representations which will be activated by that particular part. The referent of the whole description is then considered as a member of the intersection of all sets resulting from partial descriptions.

An important prerequisite of our approach is that the system explicilly represents how it has encoded information in a prescntation. Inspired by (Mackinlay, 1986), we use a relation tuple of the form:

\section{(Encodes means information context-space)}

to specify the semantic relationship between a textual or graphical means, and the information the means is to convey in a certain context space. In our approach, the third argument refers to the context space to which the encoding relation corresponds to and not to a graphical language as in Mackinlay's approach. This cuables us to use one and the same presentation means differently in different context spaces. For example, a depiction of an espresso machine may acler to an individual machine in one context space, but may scrve as a prototypical representative of an espresso machine in another. In addition, we not only specify encoding relations between individual objects, but also specify encoding relations on a generic level (c.g., that the property of being red in a picture encodes the property of being (defect in the world).

While it can be assumed that a user reads a text in scquential order, it is often not clear at which times a user looks at a picture. Therefore, it makes not always sense to further distinguish between an anaphor and its antecedent. Fortunately, our approach does not require identifying parts of a presentation as anaphora and antecedents. It suffices to recognize which parts of a description are intended to encode a uniquely determinal object. 'To express such cohesive relationships between presentation parts $\mathrm{pl}$ and $\mathrm{p} 2$, we define the predicate:

(Encodessame pl p2 c):=

(Existsw (And (Encodespl w c) (Encodes p2wc) (Forall $v$ (Implies (Or (Encodes $p 1 \vee c)$ (Encodes p $2 v \mathrm{c})$ ) $($ Coref $w v))))$

The first part of this definition expresses that there exists an object $w$ that $p 1$ and $\mathrm{p} 2$ cncode in the context space $\mathrm{c}$ while the second part means that this object $w$ is uniquely determincd.

\subsection{Links between Representations and Descriptions} In understanding a referring expression, the user has to recognize certain links between activated mental representations, between descriptions and mental representations, and between textual and graphical parts of descriptions. Which links are present in a description and which have to be inferred varies from situation to situation. To illustrate this, let's have a look at a case study carricd out in our espresso machine domain where text-picture combinations are uscd to explain how to operate an espresso machine. We assume that the user is requested to turn the temperature control of an espresso machine. In this case, identification means activating a representation the user builds up when localizing the referent in his visual field. Furthermore, we presume the user knowledge of the espresso machine as in Section 2.1; i.c., the user knows of the existence of the on/off- and the temperature control, has visual access to the two switches in the world but is not able to tell them apart. In the diagrams below, we use the abbreviations ES, $\mathrm{C}$ and $\mathrm{E}$ for the relations Encodes\$anc, Coref and Encodes respectively.

In the document fragment shown in Fig. 2, the textual refercnce expression uniquely determines a referent, but activatcs a representation $(\mathrm{t} 2 \ldots \mathrm{u})$ which docsn't contain any information to localize the referent. Conversely, the representations activated by the picture contain locative information, but here we have the problem that several object represculations are activated to the sane extent. Since only the property of being a switch, but not the property of being a temperature control is conveyed by the picture, both switch depictions become possible as antecedents of the textual referring expression.

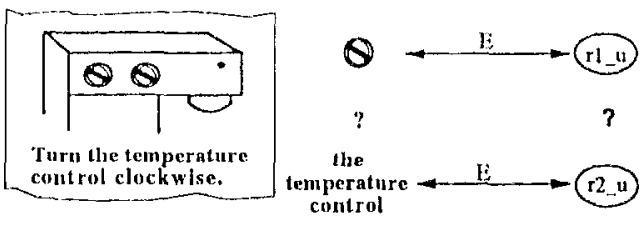

Figure 2: Missing Cohesive Link between Text and Picture

In I.ig. 3, the verbal description discriminates the referent from its altematives by altributes of the world object, namely 'being a switch', and 'being depicted in the figure' and an attribute of the depiction, namely 'being dark'. But, in contrast to the previous cxample, only one of the representations activated by the picture fits the verbal description. Thus, the user should be able to discover the anaphoric link between the verbal description and the graphical depiction and activate an appropriate representation.

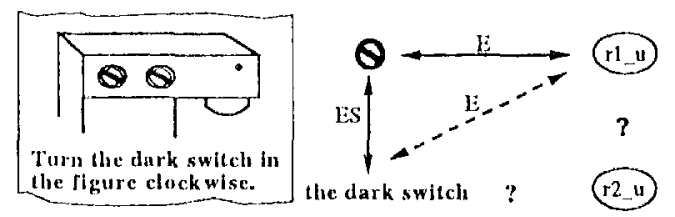

Ifigure 3: Establishing a Cohcsive Link by Incorporating Picture Attributes in Verbal Descriptions

In the previous example, an anaphoric link between text and picture has been established by including pictorial attributes in the verbal description. An alternative is to apply graphical focusing techniques as in Fig. 4. Ilere, it's very likely that the user will be able to draw a link between text and picture because he will assume that the pictorial and the textual focus coincide. This example also illustrates how the user's knowledge of the identity of objects can be curiched by means of a referring act. The verbal 
description without the graphics and the graphical depiction wilhout the text activate different representations of the switch. When considering both text and graphics, the user will conclude that they refer to the same object. 'Thus, he is not only able to identify the switch as required, he is also able to combine the different representations of the switch into onc. Note that this plicnomenon catt also be explained in terms of centering theory (Gros\% ct al., 1983). In the example, the preferred center of the picture would coincide with the backward looking enter of the text.

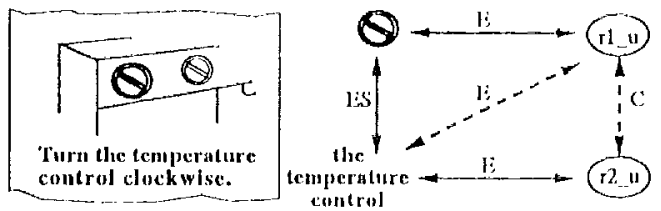

Irigure 4: Lstablishing a Colnesive liuk by Conclating Textual and Pictorial locus

The example shown in ligg. 5 differs from the previous ones in that no correspondency link between picture objects and real world objects can be established. Although the user is able to draw an anaphoric link between the verbal and the pictorial description, he is not able to visually identify the intended referent.

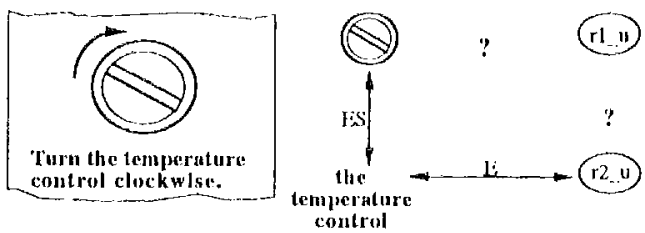

Figure 5: Missing Correspondency between Picture and World

Summing up, it can be said that a refering act is only successful when the description provides an access path to an appropriate represcntation. The user has to infer such a path from cncoding relationships and colnesive links be. tween the parts of a description. As the cxamples show, the following cases occur: a) If the user does not recognize which picture parts correspond to which world object, the referring act cither fails (ct. ling. 5) or the picture contributes nothing to its suceess. b) If the relationship between pictorial depictions and verbal descriptions is unclear, the referent can either not be found (ct. Fïg. 2) or one of the media has no influence on referent identification. c) If a graphical depiction and a verbal description activate different representations of one and the same object and the user recognizes not only these links, but also a link between the two presentation parts, he is not only able to find the referent, but also able to combine the rlifferent representations into onc (cf. ligg. 4).
3 USING TIIE MODEL, TO GLNLRATC REFERRING EXPRESSIONS

In the following, we will sketch how we have integrated the approach into the multimedia presentation system WIP (Wahlster et al., 1993). At the heart of the WIP system is a presentation planner that is responsible for determining the contents and selccting an appropriatc medium combination. The presentation planner receives as input a presentation goal (c.g., the user should know where a certain switch is located). It then tries to find a presentation strategy which matches this goal and generates a refinement-style plan in the form of a dirceled acyclic graph (DAG). This DAG reflects reflects the propositional contents of the potential document parts, the intentional goads belind the parts as well as the rhetorical relationships between them, for details sec (Andre and Rist, 1993). While the top of the presentation plan is a more or less complex presentation goal (c.g., instructing the user in switching on a device), the lowest level is formed by specifications of elementary presentation tasks (c.g., formulating a request or depicting an object). These elementaty lasks are directly forwarded to the medium-specilic generators, currently for text (Kilger, 1994) and graphics (Rist, and Andte, 1992).

The content of referring expressions is determined by the presentation planner that also decides which representattions should be activaled and which medium should be chosen for this. 'Io be able to perform these steps, we need presentation strategies for linking propositional acts with activation acts. An example of such a strategy is [1].

[1] Header: (Request S U (Action qaction) 'lext)

liffect: (BMB S U (Goal $S$ (Done U ?action)))

Applieability Conditions:

(And (Goal S (Done U ?action))

(Bel S (Complex-Operating-Action 7action))

(Bel S (Agent ?agent ?action))

(Bel S (Olject ?olject ?action)))

Main Acts:

(S-Recurest SU U

(7aclion-spec (Agent 7agent-spec) (Object ?object-spec)))

Subsidiary $\Lambda$ cts:

(Activate S U (Action 7action) ?action-spec Text)

(Activate S U ( $A$ gent ?agcnt) ?agent-spec'lext)

(Activate S U (Olject ?object) ?olject-spec Iext)

This strategy can be used to request the user to perform an action. In this strategy, two kinds of act occur: an clcmentary specch act S(urface)-Rejuest and three activation acts for specifying the action and the semantic case roles associated with the action (Activate). 'The strategy prescribes text for the subsidiary acts because the resulting refcring expressions (?action-spec, ?agent-spec and ?object-spec) are obligatory case roles of an S-Request speceh act which will be conveyed by text. For optional case roles any mediun can be taken. In addition to strategies for linking propositional and activation acts, we necd strateģics for different kinds of activation and for establishing Corct-and EncodesSame-rclationships. For cxample, strategy [2] can be used to activate a representation ?r-1 by lext and to simultancously enrich the user's knowledge 
about the identity of objects. The stratcgy only applics if there exists already an image ?pic-obj which cncodes ? $\mathrm{r}-1$, the system believes that ?r-1 and ?r-2 are representations of the same world object and if the system's model of the uscr's belicfs contains ? $1-2$. If the stratcgy is applicd, the system a) provides a unique description ?a for ? $\mathrm{r}-2$ (main act) and b) consures that the user recognizes that this description and the corresponding image specify the same object (subsidiary act).

[2] Header: (Activate S U (?cist-role ?r-1) ?d Text)

Efrect: (BMB \& U (Coref ?r-1 (1-2))

Applicability Conditions:

(And (BMB S U (Encodes ?pic-obj ?r-1 ?c))

(Bel S (Coref ? $\mathrm{r}-1$ ? $\mathrm{r}-2)$ )

(Bel S (Bcl U (Thing ? T-2))))

Main Acts:

(Provide-Unique-Description S U ? 2 ? $\mathrm{N}$ Texl)

Subsidlary Acts:

(Achieves

(BMB S U (IncodesSame ?d ?pic-ob ?c)) ?medium)

For a), we use a discrimination algorithm similat to the algorithm presented in (Reiter and Dalc, 1992). IIowevcr, we have investigated additional possibilitics for distinguishing objects from their alternatives. We can refer not only to features of an object in a scene, but also to features of the graphical model, their interpretation and to the position of picture objects within the picture, sec also (Waxinski, 1992). $\Lambda$ detailed description of our discrimination algorithm can be found in (Schnciderlöchner, 1994). 'Task b) can be accomplished by correlating the visual and the textual focus, by redundantly encoding object attributes, or by explicitly informing the user about a Coref-relationship. Such a Coref-relationship can be established by strategics for the gencration of cross-media referring expressions (as in "The icft switch in the figure is the temperature control") or by strategics for annotating objects in a figure.

\section{CONCI,USION}

We have presental a model of referring which is based on the following assumptions: 1) Mental representations of objects may be activatcd not only by textual, but also by graphical and mixed descriptions. 2) Failute and success of referring acts can be cxplained by the user's ability to tecognize certain links between these mental representations and the corresponding object descriptions. 'Lo demonstrate that the model is of practical use for the gencration of references, we have delincd presentation strategies for concept activation which serve as operators in the plan-based presentation system WIP. WIP is able to gencrate multimedia, anaphoric and cross-media referring expressions.

ACKNOWLEDGLMEN'IS: 'This work is supported by the BMI Y under grant ITW89018. We would like to thank Doug $A$ ppelt for valuable discussions and comments.

\section{RLETERNCES}

Allgayer, J., Iarbusch, K., Kobsa, A., Reddig, C., Reithinger, N. and Schmaucks, D. (1989). XIRA: A NaturalLanguage Access System to Expert Systems. Intern. Journal of Man-Machine S'tudies, 31, pp. 161-195.

Andrć, L., and Rist, T. (1993). The Design of Illustrated Documents as a Planning Task. In M.T. Maybury Ed., Intelligent Multimedia Interfaces, The MTT Press, Menlo Park, pp. 94-116.

Appclt, D., and Kronfeld, А. (1987). A Computational Model of Referring. Proc of IJCAI-8\%, pp. 640-647.

Claassen, W. (1992). Generating Referring Expressions in a Multimodal Environment. In R. Dale, E. Ilovy, D. Rösncr and O. Stock Ed., Aspects of Automated Natural Language Generation: Proc. of the 6th International Workshop on Natural Language Generation. Springer, Berlin, pp. 247-262.

Goodman, N. (1969). Languages of Art. Oxford University Press, Oxford.

Grosz, B., Joshi, A.K., and Weinstcin, S. (1983). Providing a Unified Account of Definite Noun Phrases in Discourse. Proc. of the 21st ACL, pp. 44-50.

Ilirst, G. (1981). Anaphora in Natural Language Understanding. Springer, Berlin.

Kilger, A. (1994), Using UlAGs for Incremental and Parallel Generation. Compuatational Intelligence. to appear.

Mackinlay, J. (1986). Automating the Design of Graphical Prescntations of Relational Information. ACM Transactions on Graplicis, 5(2), pp. 110-141.

McKcown, K.R., Feiner, S.K., Robin, J., Scligmann, D.D. and lanenblat, M. (1992). Generating Cross-References for Multimedia Explanation. Proc. AAAI-92, pp. 9-16.

Reiter, L., and Dale, R. (1992). A Fast Algorithm for the Generation of Referring Expressions. Proc. of COLING$92,1, \mathrm{pp} .232-238$.

Rist, 'T., and Andre (1992). From Presentation Tasks to Pictures: Towards an Approach to Automatic Graphics Design, Proc. of ECAI-92, Vienna, Austria, pp. 764768.

Schnciderlöchncr, I: (1994). Generierung von Referenzausdrücken in einem multimodalen Diskurs. Diploma 'Thesis, Universität des Saarlandes, Germany, to appear.

Stock O., and the ALIRESCO l'roject Team (1993). ALFRLSCO: Enjoying the Combination of Natural Language Processing and Hypermedia for Information Exploration. In: In M.T. Maybury Ed,, Intelligent Multimedia Interfaces, The MIT Press, Menlo Park, pp. 197-224.

Wablster, W., Andre, L., Graf, W., and Rist, T. (1991). Designing Illustrated Texts: How Language Production Is Influenced by Graphics Generation. Proc. of EACL92, Berlin, pp. 8-14.

Wahlster, W., Andre, E., Finkler, W., Profitlich, H.J., and Rist, T. (1993). Plan-Based Integration of Natural Language and Graphics Generation. AI Journal, 63, pp. $387-427$.

Wazinski, P. (1992). Generating Spatial Description for Cross-modal References. Proc. of $A N L P=92$, '[rento, lialy, pp. 56-63. 